\title{
EL RETORNO DE LA NATURALEZA: LA ÉTICA AMBIENTAL Y LA CUESTIÓN ANTROPOLÓGICA CONTEMPORÁNEA
}

\author{
Luca Valera ${ }^{1}$
}

\begin{abstract}
RESUMEN: Repensar la naturaleza en la época actual significa enfrentar las nuevas perspectivas desarrolladas por la ética ambiental, es decir, las respuestas a la crisis ecológica contemporánea. Los dos paradigmas ambientales principales, biocentrismo y antropocentrismo, antes que ser paradigmas éticos son dos visiones antropológicas desarrolladas a partir de una cierta idea del puesto del ser humano en el cosmos. En este sentido, el objeto de este artículo es destacar el hecho de que la ecología es una antropología y, debido a esto, para repensar la naturaleza tenemos que reformular nuestra visión antropológica, dada la insuficiencia a nivel teórico de los principales paradigmas ambientales.
\end{abstract}

Palabras-Clave: Antropocentrismo. Biocentrismo. Ecosistema. Crisis ecológica. Ética ambiental.

\section{INTRODUCCIÓN ${ }^{2}$}

El desarrollo actual de las investigaciones ambientales, la crisis ecológica causada por el cambio climático antropogénico (LIAO et al., 2012), el debate contemporáneo sobre la sustentabilidad (VALERA \& MARCOS, 2014), y las nuevas posibilidades imprevisibles para los seres humanos de modificar y transformar radicalmente el medio ambiente (O'BRIEN, 2012) determinaron un aumento de interés en los estudios ecológicos en los últimos años - y con ello, también en el tema de la naturaleza (VALERA, 2011). Los considerables cambios introducidos por el desarrollo tecnológico no siempre se han acompañado de una reflexión fundamentada sobre el papel de la tecnología en nuestras vidas, en la vida del medio ambiente y de los otros seres vivos. En

\footnotetext{
${ }^{1}$ Algunas de las reflexiones que se presentan en este artículo han sido previamente desarrolladas en Valera, 2014; Valera, 2017 y Valera, 2017a. Agradezco a Gabriel Vidal por su preciosa ayuda en la revisión del texto.

2 Profesor Asociado de Ética aplicada y bioética, Instituto de Filosofía, y Director del Centro de Bioética, Escuela de Medicina, Pontificia Universidad Católica de Chile, Santiago - Chile. (D) http://orcid.org/0000-0002-1693-396X E-mail: luvalera@uc.cl
}

http://doi.org/10.1590/0101-3173.2020.v43n2.10.p171

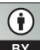

This is an open-access article distributed under the terms of the Creative Commons Attribution License. 
los últimos años, además, se ha producido un creciente interés internacional a nivel filosófico en este ámbito, dando así lugar a la emergencia de nuevas investigaciones y, en concreto, a la filosofía de la ecología y a la ética ambiental (KAWALL, 2016; VALERA, 2107). Desde el momento en que el ser humano comenzó a ser consciente de su capacidad destructiva sobre el medio ambiente también ha tratado de reflexionar sobre su relación privilegiada con los seres vivos no-humanos (JONAS, 1995).

La edad contemporánea ha conllevado, así, un interesante cambio de perspectiva: el ser humano actual ha desarrollado una conciencia más profunda acerca de su relación con el entorno y con la alteridad no humana, descubriendo al mismo tiempo la vulnerabilidad del ecosistema y de las distintas formas de vida. De esta manera, el ser humano contemporáneo vive con la conciencia de una crisis ecológica siempre inminente (BUELL, 2003), junto al conocimiento de que los recursos naturales se están agotando rápidamente (VALERA, 2019) y, por eso, está siempre en búsqueda de nuevas soluciones políticas y económicas (BECK, 1998, p. 44; p. 79-88; LEFF, 2004).

\section{El RETORNO A LA NATURALEZA: LA Ética AMBIENTAL COMO REFLEXIÓN COSMOLÓGICA Y ANTROPOLÓGICA}

Con razón, por lo tanto, la ética ambiental parece haber ganado importancia dentro del pensamiento filosófico contemporáneo: la reflexión ambiental tiene que ver con las cuestiones filosóficas de la nueva relación del ser humano con la naturaleza, ya que se refieren a la forma en que queremos vivir, al modelo de sociedad que anhelamos y a nuestra relación con otras formas de vida $^{3}$. Si quisiéramos, de hecho, definir la cuestión ambiental, podríamos decir que se trata de "un conglomerado de situaciones que, por una parte, son resultado del agravamiento de problemas relativos a las formas de uso de los recursos naturales y, por otra, de la aparición de problemas de diversa índole, como la contaminación. A su vez, estos problemas son causantes de diversos grados de disminución en la calidad de vida de la población, que en muchos casos resulta paradójica, dado el progreso alcanzado" (OJEDA \& SÁNCHEZ, 1985, p. 26).

\footnotetext{
${ }^{3}$ Retomando una valiosa idea del filósofo Adolfo Sánchez Vázquez (1984, p. 25), podríamos afirmar, de hecho, que la ética se vincula estrechamente con nuestras sociedades, ya que "la ética es la teoría o ciencia del comportamiento moral de los hombres en sociedad. O sea, es ciencia de una forma específica de conducta humana”.
} 
La ética ambiental, así, no califica como una entre las muchas éticas aplicadas que surgieron en el mundo anglosajón en la segunda mitad del siglo $\mathrm{XX}$, sino como una filosofía que tiene por objeto específico de su investigación el modo humano de habitar en el mundo ${ }^{4}$ (VALERA, 2016). Escribe Marcos (2001, p. 17):

La ética ambiental trata desde un punto de vista racional los problemas morales relacionados con el medio ambiente. Esta rama de la ética tiene cada día más importancia, dado que los problemas ambientales están hoy muy presentes, pues nuestra capacidad de intervención sobre el medio es cada vez mayor. La idea de que la ética ambiental es sencillamente ética aplicada es errónea. Para empezar porque la ética se construye desde abajo hacia arriba, desde la experiencia moral hacia los principios más abstractos, y no al revés.

En este sentido, la ética ambiental hereda la reflexión clásica sobre el tema de la naturaleza, tanto ético como ontológico, recuperando una perspectiva que fue perdida con la época moderna: la idea por la que el ser humano es una parte de la naturaleza (NÆSS, 2005, p. 222), y que, por lo tanto, no es posible separar fundamentalmente una reflexión sobre la naturaleza humana de una reflexión sobre toda la naturaleza, una reflexión antropológica de una cosmológica, y al revés (VALERA, 2017a, p. 17).

Aunque las preguntas originales de la ética ambiental se caracterizan como semánticamente densas, ya que se refieren a la constitución del ser humano mismo y a sus maneras de convivir con los otros seres vivos, muchas veces las tendencias filosóficas actuales parecen haber traicionado esta profundidad, desplazando el centro de la reflexión hacia cuestiones eminentemente ideológicas o de aplicación práctica a problemas concretos y específicos. El debate ambiental contemporáneo fluctúa, de hecho, entre dos paradigmas opuestos e irreconciliables: el antropocentrismo y el biocentrismo (o anti-antropocentrismo). Como destaca LEE (1994, p. 89-90):

Los especialistas en ética ambiental se dividen en dos líneas principales de pensamiento: el antropocentrismo/no-antropocentrismo, por un lado, y el individualismo/holismo, por el otro. Los que argumentan que no solo los seres humanos, sino también la naturaleza, tiene un valor intrínseco, rechazan el antropocentrismo, podemos decir, al menos en su forma más radical.

\footnotetext{
${ }^{4}$ En cuanto a la cuestión del habitar humano, véase: Lo stare degli uomini (Garlaschelli; Petrosino, 2012).
} 
La disputa entre los partidarios de los dos paradigmas -antropocéntrico y anti-antropocéntrico- converge, en esencia, en torno a una única pregunta fundamental: ¿Qué lugar se debe asignar al ser humano en el mundo? o, más bien ¿Cuál es la posición del ser humano en el cosmos? Si, de hecho, por un lado, hay una tendencia a ampliar los límites de la moralidad a otros seres vivos, por el otro, el universo moral gravita alrededor de la especie Homo sapiens, dándole la oportunidad de subyugar a todos los seres vivos no humanos. En este sentido el ser humano se encuentra más allá de la naturaleza, siendo un ser "antinatural", es decir, irreductible a la esfera de lo meramente natural. $\mathrm{Si}$, de hecho, el ser humano es el dueño de la naturaleza y la medida de todas las cosas, no se puede, al mismo tiempo, medirlo por la naturaleza misma (MEYER-ABICH, 1983, p. 212).

La hipótesis antropocéntrica no tiene muchos defensores en la ética ambiental, ya que se cree que el ser humano es la causa fundamental de la crisis ecológica actual: el ser humano ha explotado el ecosistema hasta el punto de haber causado daños irreparables, como el cambio climático, el agotamiento casi total de los recursos no renovables, la contaminación del aire, la pérdida de zonas verdes y la extinción de algunas especies. Por estas razones, desde los años ochenta, el pensamiento más difundido en este ámbito es sin duda el biocentrismo: "Se piensa que el anti-antropocentrismo es un punto de partida común para la ética ambiental” (SHEPPARD \& LIGHT, 2007, p. 222).

$\mathrm{Si}$, por un lado, el antropocentrismo tiende a afirmar la centralidad del ser humano en el ecosistema, es decir, su posición privilegiada dada por sus capacidades racionales, por el otro, reitera esencialmente el valor instrumental de la naturaleza, reconociendo el valor sustancialmente "antropogénico" de la misma (ROLSTON III, 1994, p. 14):

Los especialistas de filosofía moral entre los que se ocupan de ética ambiental han errado en la búsqueda de un valor en las cosas que fuera independiente de las evaluaciones humanas. Así han olvidado un punto básico acerca de la evaluación de cualquier cosa: la evaluación es siempre desde el punto de vista de un evaluador [...] Solamente los seres humanos son agentes que evalúan" (NORTON, 1991, p. 251).

La cuestión principal que divide los dos paradigmas éticos implica, entonces, una pregunta más profunda: ¿Qué determina el valor? ¿Es el ser humano él que determina el valor de la naturaleza o la naturaleza tiene valor 
en sí misma? Ese tema lleva inevitablemente a la pregunta por si el valor es intrínseco o instrumental'5.

La confusión de los niveles epistemológicos ha llevado inevitablemente a apoyar de una manera ingenua e inconsciente la "falacia naturalista": a una igual "bondad ontológica" original de cada entidad correspondería necesariamente un igual tratamiento. Sin embargo, en el debate contemporáneo inaugurado por la ética ambiental, la falacia sobre la naturaleza del valor $-\mathrm{y}$, por lo tanto, sobre el valor de la naturaleza- parece insuperable: parece esencial reconocer que hay un valor intrínseco en todos los seres vivos, cuando "los seres humanos son los metros, son los que dan valor a las cosas, incluso cuando evalúan lo que es en sí mismo" (ROLSTON III, 1994, p. 15). Si el valor parece calificarse como esencialmente "extrínseco", cada hipótesis de la existencia de un "valor intrínseco" se presentaría contradictoria: “Cuando hablamos de 'valor', siempre se indica algo que es para un destinatario. Algo tiene valor para alguien. El valor es una cualidad relacional y uno de los términos de la relación tiene que ser un 'alguien', es decir, una subjetividad capaz de evaluar. El valor es valor para la subjetividad” (VIOLA, 1997, p. 127). Si el origen del valor está, por lo tanto, necesariamente relacionado con la presencia del ser humano, capaz de "reconocer" el valor de algo que es bueno -y no tanto de "crear" un valor- la hipótesis anti-antropocéntrica resulta ser infundada, al menos en este punto: cualquier declaración de presencia de valores intrínsecos en la naturaleza, ya que requiere una persona que sitúa el valor, se califica necesariamente como una mera declaración de principios (BAIRD CALLICOTT, 1992).

Más allá de cualquier crítica de uno u otro enfoque, sin embargo, queremos destacar las raíces utilitaristas y económicas de la ética ambiental en general: el concepto de valor, que es central tanto en la especulación biocéntrica como en la antropocéntrica, se refiere necesariamente a un precio y a una misma moneda con las cuales es posible calcular todo (SCHMITT, 2010). Una vez fundado el discurso de tal manera, es difícil liberarse de las aporías que eso implica: si el valor se refiere a un precio y el precio no es un atributo original, sino que siempre se obtiene a través de un contrato, la imposibilidad de identificar valores intrínsecos es evidente en sí. Por su propia naturaleza, de hecho, el valor necesita una estimación. Y evaluar una realidad es muy distinto a describirla (VALERA \& BERTOLASO, 2016, p. 47-49).

\footnotetext{
${ }^{5}$ No tenemos la posibilidad de profundizar en este lugar el tema y por eso preferimos referirnos a otros textos (O’Neill, 1992. James, 2015, p. 64-77).
} 


\section{NATURALEZA Y SER HUMANO: EL CENTRO DE LA CUESTIÓN AMBIENTAL}

Las diferencias entre las dos matrices principales de pensamiento en la ética ambiental son diferencias metafísicas (o cosmológicas) antes que éticas: el antropocentrismo y el biocentrismo, de hecho, proponen visiones totales de la realidad completamente antitéticas. Si la filosofía antropocéntrica sigue, en esencia, la tradición metafísica occidental, la biocéntrica parece determinar un tipo de cambio de perspectiva, o una revolución copernicana; la primera destaca la persistencia y la singularidad de las entidades, reconociendo la existencia de una jerarquía ontológica entre los seres vivos a la cabeza de la cual estaría el ser humano, mientras que la segunda enfatiza la dificultad de distinguir la sustancia del proceso (el individuo del Todo), dando lugar a un doctrina del "entero como fluido" (LANZA \& BERMAN, 2013). El resultado de esta última revolución de pensamiento -que podríamos llamar “ontología de los procesos" (BRAIDOTTI, 2006, p. 199), y que constituye la base teórica de numerosas filosofías post-metafísicas contemporáneas, en primer lugar, el posthumanismo (VALERA, 2014, p. 487-489; VALERA \& ALVARADO, 2019) - es la substancialización del accidente de la relación, es decir la "web" (red), a la luz de los avances de la cibernética de Wiener. La primera consecuencia es que

[...] no podemos establecer ninguna división ontológica definitiva en el campo de la existencia. En la realidad no existe ninguna diferencia radical entre el dominio humano y el dominio no-humano [...] mientras sigamos percibiendo este tipo de fronteras no alcanzaremos a comprender qué es la conciencia ecológica profunda (FOX, 1984, p. 196).

Negando la sustancia a nivel ontológico, se rechaza también la posibilidad por la que, a nivel antropológico, el ser humano - como cualquier otro ser viviente - pueda ser considerada una entidad aislada de las otras entidades, un sujeto individual y persistente; por otro lado, además, se niega la hipótesis por la que las entidades se caracterizan como ontológicamente cerradas, y por tanto como definibles, limitadas. El biocentrismo -y con eso una cierta interpretación del ambientalismo radical (Deep Ecology) ${ }^{6}$ - se caracteriza por la eliminación de los niveles de la realidad, porque no hay una jerarquía en el ecosistema. En este sentido, el biocentrismo propone una

${ }^{6}$ Hablamos acá de una cierta interpretación de la ecología profunda: algunos ecologistas profundos piensan que la obra de Næss sea el primer paso para desarrollar una visión más holista del ser humano, subrayando la prioridad -a nivel ético también- del Todo por sobre el ser humano. El pensamiento de Næss, todavía, es más complejo y no llegaría consistentemente a esta conclusión (Valera, 2015, p. 28-33). 
interpretación autónoma de la inmanencia de Spinoza (NÆSS, 1977, p. 46) -que le permite superar "la dualidad de valores espíritu/materia, alma/ cuerpo", porque la Naturaleza ahora coincide con la res extensa y la res cogitans, al mismo tiempo- distanciándose así de la explotación y el empobrecimiento moderno de lo material, rendido inerte y sujeto a la supremacía del espíritu. $\mathrm{Si}$, últimamente, lo que existe es solo la Naturaleza y las entidades singulares no son más que la simple reverberación contingente de esa, no tiene sentido establecer la existencia de "una jerarquía moral del mundo predeterminada" (NÆSS, 1977, p. 50).

Se puede reinterpretar la naturaleza a la luz del panteísmo inmanentista implícito en el spinozismo del biocentrismo: esto permite asignar un valor "intrínseco" y una prioridad a las entidades súper-individuales (como el ecosistema). Meyer-Abich (1984, p. 90, p. 100, p. 187) escribe:

La naturaleza continúa en nosotros haciéndose lenguaje y arte, así como continúa en el resto de los seres vivos a partir del momento del que viven, por así decirlo, su vida (es decir, la vida de la naturaleza). Nuestras vidas y la vida del mundo que nos rodea son su vida. [...] La natura naturans, la fuerza creativa, coincide en todos los lugares con todo: así que éste es el verdadero centro del mundo. [...] Que un árbol muera o que muera un hombre, en ambos casos, un ser vivo muere y regresa a la Tierra.

Una vez aceptada esta inmanencia, la siguiente medida tomada por el biocentrismo es el antropomorfismo del ecosistema; esto permite también considerar la naturaleza como un "súper-organismo", es decir, como una entidad con su propia "conciencia" y "voluntad", de modo que el ser humano no es nada más que una pequeńa parte carcinógena, como Potter (2002, p. 123-124) ha recordado:

‘En lo que concierne a la naturaleza, nosotros somos como un cáncer cuyas células extrańas se multiplican sin control, descaradamente exigiendo el alimento que todo el cuerpo necesita [...]'. En otras palabras, podemos hacernos la pregunta, ¿es el destino del hombre ser para la tierra viviente lo que el cáncer es para el hombre?

El ser humano, por ende, ha perdido su posición central en el universo, convirtiéndose en una de las muchas realidades en el sistema y 
dando cumplimiento así al igualitarismo biosférico ${ }^{7}$. De hecho, se convierte precisamente en la célula más peligrosa del sistema, la más destructiva, capaz de innumerables eco-desastres y, por lo tanto, a erradicar. El principio detrás de este razonamiento es muy sencillo e intuitivo: el sistema general (o Ecosistema/ Gaia) tiende a la propia conservación y necesita de los subsistemas individuales solamente como medio para lograr este único fin. Como súper-organismo vivo, por lo tanto, Gaia "decide" (también de la vida de los subsistemas) y el ser humano sólo puede aceptar.

Hay, de hecho, en la concepción biocéntrica una estrecha relación entre la subjetividad humana y el ecosistema o, mejor dicho, ya no es posible distinguir el "yo" que percibe y el "mundo natural" como objeto de esa misma percepción. El resultado de un tal anti-dualismo es la representación del sujeto como una entidad sin límites, extendida y constituida por las interacciones que se producen en el ecosistema: "La naturaleza es una unidad, un todo, un subjeto, y el "yo" (en el sentido tanto físico como mental) no es sólo una continuación de esta, sino que está constituido por ella. Yo y la naturaleza somos integrados conceptual y metafísicamente" (BAIRD CALLICOTT, 1985, p. 273-274).

La integración total entre el ser humano y la naturaleza, dirigida al rescate del individuo de su aislamiento, es la consecuencia de la anulación del límite que necesariamente da lugar a la identidad (VALERA, 2015a, p. 1457-1461): para que el ser humano se inscriba en la naturaleza, no debe tener barreras estructurales que delinean precisamente sus límites de vida. Si el ser humano se caracteriza por ser una entidad esencialmente no-natural (debida a su facultad racional, su libertad, su autoconciencia, etc.), no se podría llevar a cabo la hibridación predicada por el biocentrismo: la reducción del ser humano a homo naturalis es necesaria para una integración completa. La apertura moral del homo (totaliter) naturalis, por lo tanto, coincide con una abertura total a nivel ontológico: el ser humano coincide con la Naturaleza, ya que no es ontológicamente "impermeable" a esta (no tiene, en definitiva, límites) y no está cerrado porque su naturaleza coincide con todas sus relaciones.

El hecho de que los cuerpos no estén cerrados -concepto central en la especulación ecologista- se expresa como la necesidad de redefinir los seres humanos como meros entes biológicos, formados por flujos vitales que se

\footnotetext{
${ }^{7}$ Para entender la relación entre parte y todo -o entre individuo y sistema- es útil referirse a la "Teoría general de los sistemas" desarrollada por von Bertalanffy (2006).
} 
extienden más allá del límite del cuerpo ${ }^{8}$. La condición de posibilidad del biocentrismo es la permeabilidad de los individuos a los procesos que siempre lo preceden: en esencia, es el concepto de vida que apoya estos procesos. El "cuerpo ecológico" sin límites se origina a partir de una materia que no tiene limitaciones espaciales, temporales o de forma, por lo que no hay nada en realidad externo al cuerpo, ya que no es posible marcar con precisión los límites reales del cuerpo mismo. De manera que, una vez eliminados los límites del cuerpo y reducida la corporeidad a mera función, esa se convierte en una ficción inútil y, por lo tanto, completamente reemplazable. La consecuencia inmediata es que los cuerpos, en el sentido de entidades aisladas y separadas, existen sólo como fruto del pensamiento: no hay nada verdaderamente independiente o separado del contexto vital en el que el cuerpo mismo se inserta.

\section{REPENSAR LA NATURALEZA}

Si el antropocentrismo tiende a perder el sentido de la integridad y de las realidades complejas y "emergentes" como el ecosistema, el biocentrismo, por el contrario, "disuelve no solo la noción del ser humano como distinto del medio ambiente, sino también la noción del mundo como compuesto por 'entidades' definidas y compactas, separadas" (FOX, 1984, p. 194). La dificultad de ir más allá de estos paradigmas incomunicables (y que, al mismo tiempo, ofrecen visiones igualmente reduccionistas e incompletas), es probablemente determinada por una concepción parcial de la naturaleza y por una idea pobre del ser humano. En este sentido, la cosmología desarrollada por los dos paradigmas parece ser hija legítima de una bien definida antropología: la idea del ser humano afecta, así, también a la idea de la naturaleza. Si en el biocentrismo, de hecho, la naturaleza se obtiene a través de una operación de antropomorfización de la realidad sobre-individual (es decir, Gaia, el ecosistema, etc.), en la opción antropocéntrica el medio ambiente (o el conjunto de los seres vivos no-humanos) se caracteriza por una substracción en comparación con el ser humano: cada ser vivo que no tiene las características superiores del ser humano se puede calificar como "natural".

Una vez más, entonces, es el lugar del ser humano en el cosmos a delinear sus posibilidades de acción y movimiento: por un lado, la consideración del ser humano como totalmente natural determina la homogeneidad de los vivientes

\footnotetext{
${ }^{8}$ Otra opción, claramente, es la contraria, es decir, atribuirle a la naturaleza las mismas cualidades que atribuimos a nosotros (racionalidad, conciencia de sí, etc.).
} 
y, por lo tanto, la hegemonía (inclusive a nivel ético) de las realidades sobreindividuales (biocentrismo); por el otro, la posición del ser humano fuera del ámbito de lo natural establece una jerarquía ontológica entre los seres vivos y, por ende, permite al ser humano actuar de acuerdo con su superioridad (antropocentrismo). Se deduce así que la posibilidad de intervención humana en la naturaleza se determina a partir del papel que él asume en la naturaleza: el comportamiento humano (la no interferencia o el uso/gestión de los recursos naturales) tiene siempre que ver con la esencia humana misma (¿Es el ser humano "el cáncer del planeta" o su dueño?).

Solo a partir de este último punto de vista, entonces, podemos hablar de un uso correcto de ciertas tecnologías. Las posiciones ideológicas de la tecnofobia y de la tecnofilia (BUNGE, 1995, p. 95) serían, por ende, el resultado no deseado de una concepción "pobre" y unidimensional del ser humano: si, por un lado, se rechaza todo lo que es técnico (tecnofobia), a la luz de un ideal encantado de pureza a-técnica, por el otro (tecnofilia) se concibe la técnica como medio necesario para el encuentro entre la naturaleza y el ser humano, que es constitucionalmente deficiente a nivel instintivo. Ambas opciones surgen de supuestos aceptados acríticamente, es decir, de una evaluación a priori positiva del progreso y, por otra parte, de un miedo instintivo determinado por una capacidad humana necesariamente destructiva.

La reflexión de la ética ambiental, entre las muchas ventajas y un buen número de vacíos teóricos, tiene el mérito de haber enfocado de nuevo el debate filosófico contemporáneo en el tema de la naturaleza, emancipándola de las especulaciones de la filosofía occidental y resolviendo el problema de considerarlo como un concepto demasiado lleno de significados diferentes y por eso incomprensible. Así Attfield (1994, p. 45) describe la necesidad de un replanteamiento del concepto de naturaleza:

Para empezar, necesitamos un claro significado de la expresión 'naturaleza' [...]. Por eso, cuando hablamos de una recuperación de la naturaleza, el término 'naturaleza' no se puede utilizar en el primer sentido desarrollado por Mill: 'Todo lo que es - las capacidades y las propiedades de todas las cosas'. Así que en ese sentido no hay posibilidad de que la naturaleza sea destruida o dańada, y mucho menos reconstruida. El segundo significado de 'naturaleza' descrito por Mill, sin embargo, es más relevante: 'Lo que se lleva a cabo sin la intervención humana voluntaria e intencional'. La naturaleza (en este sentido), obviamente, puede modificarse mediante la actividad humana. Además, hay una dificultad adicional en la posibilidad de restaurarla: ¿Cómo puede algo, cuya esencia es independiente del ser humano, ser reconstruida por la actividad humana? 
La ética ambiental, motivada por la crisis ecológica, vuelve así a considerar a la naturaleza como un mero conjunto de los vivientes, liberada por la aparente pesadez de las especulaciones filosóficas occidentales. A través de la eliminación de las implicaciones metafísicas de los vivientes, y equiparando los entes a la luz de una misma unidad de medida (es decir, los procesos biológicos), se hace posible diseñar un sistema autosuficiente y totalmente inmanente: se reemplaza así el concepto de naturaleza con el concepto de ecosistema, que tiene una connotación semántica de menor densidad filosófica y que está inmediatamente relacionado a la idea de estructura compleja.

Si quisiéramos reconstruir históricamente la génesis de este fenómeno, deberíamos considerar por lo menos dos grandes momentos que han entregado dos interpretaciones contrastantes de la naturaleza: la edad antigua y la moderna. En la antigüedad la naturaleza era, de hecho, lo sagrado, la esencia de la divinidad, y por lo tanto era lo venerable, pero al mismo tiempo intangible, porque era el espejo de algo trascendente y misterioso. Así Chesterton (1988, p. 93) describe las dos actitudes hacia la naturaleza:

Los poetas menores de nuestro tiempo son naturalistas, y hablan del arbusto y del arroyo; pero los cantores de las viejas fabulas y epopeyas estaban por lo sobrenatural, y hablaban del dios del arroyo y del dios del arbusto. A esto se refieren los hombres de hoy cuando dicen que los antiguos no 'apreciaban la Naturaleza', porque la suponían divina. Las niñeras no hablan a los niños de la hierba del campo, sino de los espíritus que danzan sobre ella, así como los arcaicos griegos no veían árboles, sino dríadas.

A través de las investigaciones de la modernidad, la naturaleza se convierte, en cambio, en el universo infinito e indefinido, reducible a objeto material y, por lo tanto, comprensible. La naturaleza medieval como elemento normativo se transforma, en la época moderna, en el mundo sencillo de la existencia que procede de forma indefinida, y por lo tanto puede ser modelado por el único ser con autonomía creacional, es decir, por el ser humano. La intangibilidad de la naturaleza- que está en las raíces de la idea de custodia del ser humano antiguo y medieval- se convierte así en la total disponibilidad en la edad moderna y, aún más, en la inconsistencia completa en el mundo contemporáneo, de modo de que la naturaleza no es más considerada como algo intacto, no perturbado por la violencia del ser humano: de esta manera el alcance de lo que es natural y original tiende a disminuir gradualmente en el mundo contemporáneo. Se lleva a cabo el proyecto baconiano y cartesiano 
de dominio (poder) sobre el mundo natural a través del conocimiento de las estructuras latentes en ello (saber). Como escribe Ferry (1994, p. 122):

Desde Descartes y su formidable proyecto de dominación, no habríamos dejado de dominar al mundo inmoderadamente. Primero, lo privamos de todo misterio al decretarlo manipulable y calculable a porfía. Se acabaron el animismo y las cualidades ocultas, esas fuerzas misteriosas que todavía atravesaban la naturaleza de los alquimistas de la Edad Media. Pero hay más: no nos bastó desencantar al universo, e instauramos, con el nacimiento de la industria moderna, los medios para consumirlo hasta su total agotamiento.

La actitud moderna de dominación de la naturaleza esconde, por otra parte, una tendencia opuesta y complementaria a esa actitud, muy presente hoy en día como ya en la era moderna: el espiritualismo. Escribe Allodi (2009, p. 135), en un comentario a la obra "Rousseau. Ciudadano sin patria" de Spaemann:

El pensamiento filosófico moderno, basándose en un proceso que se puede llamar 'inversión de la teleología' o 'de-teleologización', aparece caracterizado por una dialéctica irresoluble entre naturalismo y espiritualismo: un naturalismo por lo que la naturaleza no tiene más una actitud espiritual, y un espiritualismo por lo que el espíritu no tiene más una dimensión natural. Se trata de una dialéctica de dos reduccionismos que se refieren continuamente el uno al otro.

\section{ConsideraCiONES CONCLUSIVAS: Los DESAFÍOS “ANTROPOLÓgICOS” PENDIENTES DE LA ÉTICA AMBIENTAL}

La relación del ser humano con la naturaleza sigue una dinámica doble y contraria, en la historia del pensamiento: el ser humano fluctúa regularmente entre ser asimilado en la naturaleza y un sentido de independencia que lo ha llevado a rebelarse contra la naturaleza misma. El intento del ser humano moderno y contemporáneo de emerger de la esfera de lo natural es, pues, el principal síntoma de esta conciencia de ser asimilado al mundo; al mismo tiempo, su querer reconocerse sólo como un animal entre otros animales es el reverso de la medalla. El retorno actual a una forma de "panteísmo naturalista" encuentra, por lo tanto, una justificación racional: una vez vaciada la naturaleza de sus vínculos con la esencia de las cosas, una vez asumidas sin crítica las teorías evolucionistas y recuperada la doctrina sistémica a la luz de la cibernética, las categorías de fin y creación parecen inútiles: "No existe 
un orden moral del mundo previo. La justicia humana no es una ley de la naturaleza” (NÆSS, 2005, p. 387).

La idea de finalidad intrínseca en la naturaleza y la constante referencia a principios trascendentes se vuelven así obsoletas a causa de la introducción de la idea de un sistema ecológico homeostático y causalmente cerrado (CAPRA, 1998). El ecosistema se presenta ahora como una forma actualizada del concepto de naturaleza, sin más referencias explícitas ni trascendentes y regulado por procesos causales de retroalimentación circular y de homeostasis.

En este contexto de replanteamiento de la idea de naturaleza, el paradigma antropocéntrico parece anticuado en cuanto es incapaz, con sus medios éticos y epistemológicos, de resolver las dificultades creadas por el ser humano mismo y de hacer frente a un cambio tan radical:

La situación actual es de indiferencia y de pobreza: el Dios trascendente se ha ido, y la naturaleza ha sido despojada de cualquier atributo que podría promover una reverencia profunda y fructífera interacción personal. [...] Lo que queda de la naturaleza es vista como un material para satisfacer las necesidades humanas. [...] La naturaleza es considerada como algo neutral u hostil, y ha sido en gran medida, pero no completamente, subyugada y conquistada (N/ESS, 2005, p. 382).

Por otro lado, la sabiduría holística y la "visión total" del biocentrismo parecen ser capaces de acercar al ser humano a la naturaleza a través de la reconstrucción de la circularidad del ego y del mundo exterior (CAPRA, 1998, p. 25-34). Pero, al hacerlo, el ser humano podría perderse en las infinitas redes que rebasan los límites de su lábil cuerpo, consiguiendo sin duda una integración con el Todo, pero perdiendo su identidad.

El desafío lanzado por la ética ambiental es, entonces, al mismo tiempo antropológico, moral y metafísico/cosmológico: la posición del ser humano en el cosmos (en el centro o en una periferia remota del universo) sugiere también su papel y la consecuente idea de naturaleza. Por otro lado, sin embargo, las cuestiones esenciales planteadas por los dos paradigmas (“¿Cuál es la posición del ser humano en el cosmos?”, “¿Cómo el ser humano habita el planeta?"), permanecen sin resolverse, ya que se desarrollan a partir de dos modelos antropológicos incompletos e ideológicos. Quizás, la ética ambiental debería proponer una visión antropológica más completa y compleja, para volver a replantear el rol del ser humano en la naturaleza. En este sentido, 
parece adecuado afirmar que la visión de la naturaleza depende de la visión antropológica, ya que la ecología es una antropología (VALERA, 2013, p. 44).

VALERA, L. Coming back to nature: environmental ethics and the contemporary anthropological issue. Trans/form/ação, Marília, v. 43, n. 2, p. 171-188, Abr./Jun., 2020.

\begin{abstract}
Rethinking nature nowadays means to face the new perspectives developed by environmental ethics, i.e., the responses to the contemporary ecological crisis. The two main environmental paradigms, biocentrism and anthropocentrism, even before being ethical paradigms, are two anthropological visions developed from a certain idea of the position of human beings in the cosmos. In this sense, the purpose of this article is to highlight the fact the ecology is rooted in anthropology. In order to rethink the idea of nature, then, we have to reformulate our anthropological view, given the failure of the two main environmental paradigms at this theoretic level.
\end{abstract}

KEYWORDS: Anthropocentrism. Biocentrism. Ecosystem. Ecological crisis. Environmental ethics.

\title{
REFERENCIAS
}

ALLODI, L. Postfazione. Robert Spaemann: la filosofia come educazione alla realtà. In: SPAEMANN, R. Rousseau: cittadino senza patria. Dalla "polis alla natura". Milano: Ares, 2009, p. 133-157.

ATTFIELD, R. Rehabilitating Nature and Making Nature Habitable. In: ATTFIELD, R; BELSEY, A. (eds.). Philosophy and the Natural Environment. Cambridge: Cambridge University Press, 1994, p. 45-58.

BAIRD CALLICOTT, J. Intrinsic Value, Quantum Theory, and Environmental Ethics. Environmental Ethics, v. 7, p. 257-275, 1985.

BAIRD CALLICOTT, J. Rolston on Intrinsic Value: A Deconstruction. Environmental Ethics, v. 14, p. 129-143, 1992.

BECK, U. La sociedad del riesgo. Hacia una nueva modernidad. Barcelona: Paidós, 1998.

von BERTALANFFY, L. Teoría general de los sistemas: fundamentos, desarrollo, aplicaciones. Ciudad de México: Fondo de Cultura Económica, 2006.

BRAIDOTTI, R. Posthuman, all too human: Towards a new process ontology. Theory, Culture \& Society, v. 23, n. 7-8, p. 197-208, 2006.

BUELL, F. From Apocalypse to Way of Life. Environmental Crisis in the American Century. New York: Routledge, 2003. 
BUNGE, M. Sistemas sociales y filosofía. Buenos Aires: Editorial Sudamericana, 1995.

CAPRA, F. La trama de la vida. Una nueva perspectiva de los sistemas vivos. Barcelona: Editorial Anagrama, 1998.

CHESTERTON, G.K. Ortodoxia. Barcelona: Alta Fulla, 1988.

FERRY, L. El nuevo orden ecológico. El árbol, el animal, el hombre, Barcelona: Tusquets editores, 1994.

FOX, W. Deep Ecology: A New Philosophy of our Time? The Ecologist, v. 14, n. 5-6, p. 194-200, 1984.

GARLASCHELLI, E.; PETROSINO, S. Lo stare degli uomini. Sul senso dell'abitare e sul suo dramma. Milano: Marietti 1820, 2012.

JAMES, S.P. Environmental Philosophy. An Introduction. Cambridge: Polity Press, 2015.

JONAS, H. El principio de responsabilidad. Ensayo de una ética para la civilización tecnológica. Barcelona: Herder, 1995.

KAWALL, J. A History of Environmental Ethics. In: GARDINER, S.M.; THOMPSON, A. (eds.). The Oxford Handbook of Environmental Ethics. New York: Oxford University Press, 2016.

LANZA, R.; BERMAN, R. Biocentrism. How life and Consciousness are the Keys to Understanding the True Nature of the Universe. Dallas: Benbella, 2013.

LEE, K. Awe and Humility: Intrinsic Value in Nature. Beyond an Earthbound Environmental Ethics. In: ATTFIELD, R.; BELSEY A. (eds.). Philosophy and the Natural Environment. Cambridge: Cambridge University Press, p. 89-102, 1994.

LEFF, E. Racionalidad ambiental. La reapropiación social de la naturaleza. Buenos Aires: siglo xxi editores, 2004.

LIAO, S.M.; SANDBERG, A.; ROACHE, R. Human Engineering and Climate Change. Ethics, Policy \& Environment, v. 15, n. 2, p. 206-221, 2012.

MARCOS, A. Ética ambiental. Valladolid: Universidad de Valladolid, 2001.

MEYER-ABICH, K.M. Wege zum Frieden mit der Natur: Praktische Naturphilosophie für die Umweltpolitik. Munich: C. Hanser, 1984.

MEYER-ABICH, K.M. What Sort of Technology Permits the Language of Nature? Conditions for Controlling Nature-Domination Constitutionally. In: DURBIN, P.T.; RAPP, F. (eds.). Philosophy and Technology. Dordrecht: D. Reidel, p. 211-234, 1983.

NÆSS, A. Spinoza and Ecology. Philosophia, v. 7, n. 1, p. 45-54, 1977.

NÆSS, A. The Three Great Movements. In: GLASSER, H.; DRENGSON, A. (eds.). The Selected Works of Arne Nass. Vol. X. Dordrecht: Springer, p. 219-225, 2005. 
NORTON, B. G. Toward unity among environmentalists. New York: Oxford University Press, 1991.

O'BRIEN, K. Global environmental change II: From adaptation to deliberate transformation. Progress in Human Geography, v. 36, n. 5, p. 667-676, 2012.

O'NEILL, J. The varieties of intrinsic value. The Monist. v. 75, n. 2, p. 119-137, 1992.

OJEDA, O.; SÁNCHEZ, V. La cuestión ambiental y la articulación sociedad-naturaleza. Estudios Sociológicos, v. 3, n. 7, p. 25-46, 1985.

POTTER, V.R. Bioética. La ciencia de la supervivencia. Selecciones de bioética, v. 1, p. 121-139, 2002.

ROLSTON III, H. Value in Nature and the Nature of Value. In: ATTFIELD, R.; BELSEY, A. (eds.). Philosophy and the Natural Environment, Cambridge: Cambridge University Press, p. 13-20, 1994.

SÁNCHEZ VÁSQUEZ, A. Ética. Barcelona: Editorial Crítica, 1984.

SCHMITT, C. La tiranía de los valores. Buenos Aires: Hydra, 2010.

SHEPPARD, J.W.; LIGHT, A. Rolston on Urban Environments. In: PRESTON, C.J.; OUDERKIRK, W. (eds.). Nature, Value, Duty. Life on Earth with Holmes Rolston, III. Dordrecht: Springer, p. 221-236, 2007.

VAlERA, L., ALVARADO, J.T. Posthumanismo e Hibridación. Pensamiento. Revista de Investigación e Información Filosófica, v. 75, n. 283, p. 307-319, 2019.

VALERA, L.; BERTOLASO, M. Understanding Biodiversity from a Relational Viewpoint. Tópicos, Revista de Filosofía, v. 51, p. 37-54, 2016.

VALERA, L.; MARCOS, A. Desarrollo humano sostenible: una visión aristotélica. Isegoría. Revista de filosofía moral y política, v. 51, p. 671-690, 2014.

VALERA, L. Arne Næss. Introduzione all'ecologia. Pisa: ETS, 2015.

VALERA, L. Ecologia ed ecologie. Medicina nei secoli. Arte e scienza, v. 23, n. 3, p. 1015-1044, 2011.

VALERA, L. Ecología humana. Nuevos desafíos para la ecología y la filosofía. Arbor. Ciencia, Pensamiento y Cultura, v. 195, n. 792, a509, 2019.

VALERA, L. Ecologia umana. Le sfide etiche del rapporto uomo/ambiente. Roma: Aracne, 2013.

VALERA, L. Un nuovo cancro per il pianeta? Natura, ambiente ed essere umano nell'etica ambientale contemporanea. Teoria. Rivista di filosofia, v. 2, p. 175-192, 2014.

VALERA, L. Introducción. ¿Qué es la ética ambiental? Desde sus raíces hacia el futuro. Cuadernos de Bioética, v. XXVII, n. 3, p. 289-292, 2016. 
VALERA, L. La dimensión religiosa de la ecología. La Ecología Profunda como paradigma. Teología y vida, v. 58, n. 4, p. 399-419, 2017.

VALERA, L. La necesaria presencia del ser humano en una verdadera perspectiva ecológica: bases antropológicas para una ecología humana. In: GOMES ALVIM, R.; MARQUES, J. (eds.). As raízes da Ecologia Humana. Maceió: SABEH, p. 17-33, 2017a.

VALERA, L. Post-Humanism: Still or Beyond Humanism. Cuadernos de Bioética, v. 25, n. 3, p. 481-491, 2014.

VALERA, L. Tecnologia ed ecologia. Dall'etica alla metafisica, dalla negazione del limite alla negazione dell'uomo. Pensamiento, v. 71, n. 269, p. 1453-1462, 2015 a.

VIOLA, F. Dalla natura ai diritti. I luoghi dell'etica contemporanea. Roma-Bari:

Laterza, 1997.

Recebido: 06/7/2016

Aceito: 30/6/2019 
VALERA, L. 